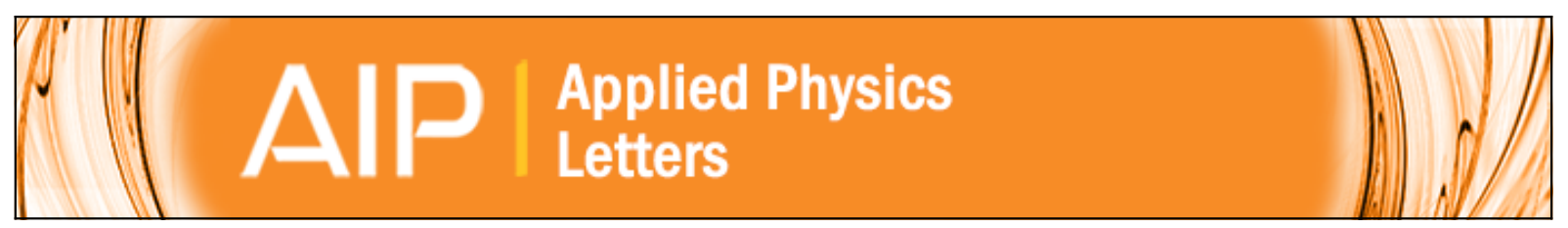

Two resistive switching regimes in thin film manganite memory devices on silicon

D. Rubi, F. Tesler, I. Alposta, A. Kalstein, N. Ghenzi, F. Gomez-Marlasca, M. Rozenberg, and P. Levy

Citation: Applied Physics Letters 103, 163506 (2013); doi: 10.1063/1.4826484

View online: http://dx.doi.org/10.1063/1.4826484

View Table of Contents: http://scitation.aip.org/content/aip/journal/apl/103/16?ver=pdfcov

Published by the AIP Publishing

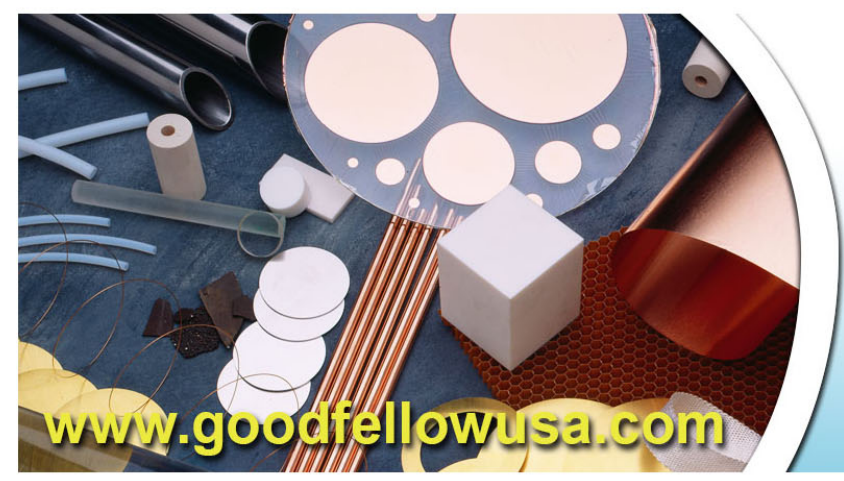

BOOdFEl/III

metals • ceramics $\cdot$ polymers

composites $\bullet$ compounds • glasses

Save $5 \%$ • Buy online 70,000 products $\cdot$ Fast shipping 


\title{
Two resistive switching regimes in thin film manganite memory devices on silicon
}

\author{
D. Rubi, ${ }^{1,2,3, a), b)}$ F. Tesler, ${ }^{1,2, b)}$ I. Alposta, ${ }^{1}$ A. Kalstein,,${ }^{1,2}$ N. Ghenzi, ${ }^{1,2}$ F. Gomez-Marlasca, ${ }^{1}$ \\ M. Rozenberg, ${ }^{2,4,5}$ and P. Levy ${ }^{1,2}$ \\ ${ }^{1}$ Centro Atómico Constituyentes (CNEA), San Martín, Buenos Aires, Argentina \\ ${ }^{2}$ Consejo Nacional de Investigaciones Científicas y Técnicas (CONICET), Buenos Aires, Argentina \\ ${ }^{3}$ Escuela de Ciencia y Tecnología, UNSAM, San Martín, Buenos Aires, Argentina \\ ${ }^{4}$ Laboratoire de Physique des Solides, UMR 8502, Université Paris-Sud XI, Orsay 91405, France \\ ${ }^{5}$ Departamento de Física J. J. Giambiagi, FCEN, Universidad de Buenos Aires, Ciudad Universitaria Pab. 1, \\ 1428 Buenos Aires, Argentina
}

(Received 21 August 2013; accepted 6 October 2013; published online 18 October 2013)

\begin{abstract}
Bipolar resistive switching in low cost $\mathrm{n}-\mathrm{Si} / \mathrm{La}_{2 / 3} \mathrm{Ca}_{1 / 3} \mathrm{MnO}_{3} / \mathrm{M}(\mathrm{M}=\mathrm{Ti}+\mathrm{Cu})$ devices was investigated. For low SET compliance currents (CC), an interfacial-related resistive switching mechanism, associated to the migration of oxygen vacancies close to the manganite/metal interface, is operative. Simulations using the voltage enhanced oxygen vacancies drift model validate our experimental results. When further increasing the $\mathrm{CC}$, we have observed the onset of a second, filamentary, resistive switching regime with a concomitant collapse of the ON/OFF ratio. We finally demonstrate that it is possible to delay the onset of the filamentary regime by controlling the film thickness. (C) 2013 AIP Publishing LLC. [http://dx.doi.org/10.1063/1.4826484]
\end{abstract}

The electric-pulse-induced resistance switching (RS) effect $^{1,2}$ has been extensively studied in the past years due to the possibility of developing resistance random access memories (RRAM), which remains a strong candidate for next generation of non-volatile memories. RRAM technology is very attractive due to its simple metal/oxide/metal structure, high writing/erasing speed, high storage density and low power consumption. ${ }^{3}$ Although different models have been proposed to explain the RS behavior, such as trap controlled spacecharge-limited-current conduction, ${ }^{4}$ electrochemical migration of oxygen, ${ }^{5,6}$ oxidation/reduction reaction, ${ }^{7}$ oxygen-vacancy driven correlation effects, ${ }^{8}$ and formation/rupture of conducting nanofilaments, ${ }^{9}$ the physics behind the RS mechanism has not been completely elucidated so far. The celebrated magnetoresistive manganites, ${ }^{10,11}$ from the initial report by Ignatiev et al., ${ }^{12}$ also displayed RS effects both in ceramics and thin films. ${ }^{13-16}$ Most papers reporting RS in manganite thin films deal with epitaxial layers ${ }^{17,18}$ or polycrystalline films grown on platinized silicon; ${ }^{4,19}$ here we show that a reliable RS behavior can be obtained by growing $\mathrm{La}_{2 / 3} \mathrm{Ca}_{1 / 3} \mathrm{MnO}_{3}$ directly on n-type silicon, which is a low-cost option that could also facilitate device integration with standard electronics. We obtained ON/OFF ratios up to $\sim 400$ and good reproducibility of the electric response. Finally, we demonstrate the change from an interfacial-related RS into a filamentary regime, which is controlled by the SET compliance current.

We grew $100 \mathrm{~nm} \mathrm{La} \mathrm{La}_{2 / 3} \mathrm{Ca}_{1 / 3} \mathrm{MnO}_{3}$ (LCMO) manganite thin films by pulsed laser deposition (pulsed Q-switched Spectra Physics Laser with $\lambda=355 \mathrm{~nm}$ and a repetition rate of $10 \mathrm{~Hz}$ ) at an oxygen pressure of 0.13 mbar and a temperature of $680^{\circ} \mathrm{C}$. Films were grown on top of highly conductive n-type silicon $(\rho<0.005 \Omega \mathrm{cm})$, which also acted as

\footnotetext{
a) Author to whom correspondence should be addressed. Electronic mail: rubi@tandar.cnea.gov.ar

${ }^{b} \mathrm{D}$. Rubi and F. Tesler contributed equally to this work.
}

bottom electrode. We used as top electrode a bilayer of $\mathrm{Ti}$ $(10 \mathrm{~nm})$ and $\mathrm{Cu}(100 \mathrm{~nm})$, fabricated by sputtering and shaped by means of standard optical lithography. The top electrode areas ranged between $0.049 \mathrm{~mm}^{2}$ and $0.785 \mathrm{~mm}^{2}$. The films thickness was estimated by cross-view scanning electron microscopy. X-ray diffraction showed that the films resulted single phase and polycrystalline. ${ }^{20} \mathrm{X}$-ray photoemission spectroscopy (XPS) suggests that the Mn valence is +2.7 , indicating an oxygen stochiometry of $\sim 2.68$. This oxygen deficiency was previously shown to improve the electrical performance of the devices. ${ }^{21}$ The electrical characterization was performed with a Keithley 2612 SMU hooked to a probe station. The acquisition software was programmed on the Labview environment.

We have recorded simultaneously, at room temperature, pulsed I-V curves and Hysteresis Switching Loops (HSL). ${ }^{5}$ The pulsed I-V curve consists on applying a sequence of voltage pulses of different amplitudes $(0 \rightarrow 8 \mathrm{~V} \rightarrow-8 \mathrm{~V} \rightarrow 0$, with a time-width of a few milliseconds and a step of $50 \mathrm{mV}$ ) while the current is measured during the application of the pulse. We recall that this is a dynamic measurement. Additionally, after the application of each of these pulses we apply a small reading voltage of $100 \mathrm{mV}$ that allows us to measure the current and evaluate the remnant resistance state HSL. Figure 1(a) displays the electrode configuration and polarities used for the electrical measurement. Figure 1(b) shows the dynamic I-V curve corresponding to one of the samples. The sample is initially in a High Resistance State (HRS) and remains in this state until a positive voltage of $\sim 6 \mathrm{~V}$ is applied, when there is a sudden transition to a Low Resistance State (LRS, SET process). A Compliance Current (CC) of $30 \mathrm{~mA}$ is externally programmed to avoid device damage during the SET process. When the voltage is decreased, the system remains in the low resistance state until a negative voltage of $\sim-2.5 \mathrm{~V}$ is reached, when there is a current drop reflecting the transition from low to high resistance 

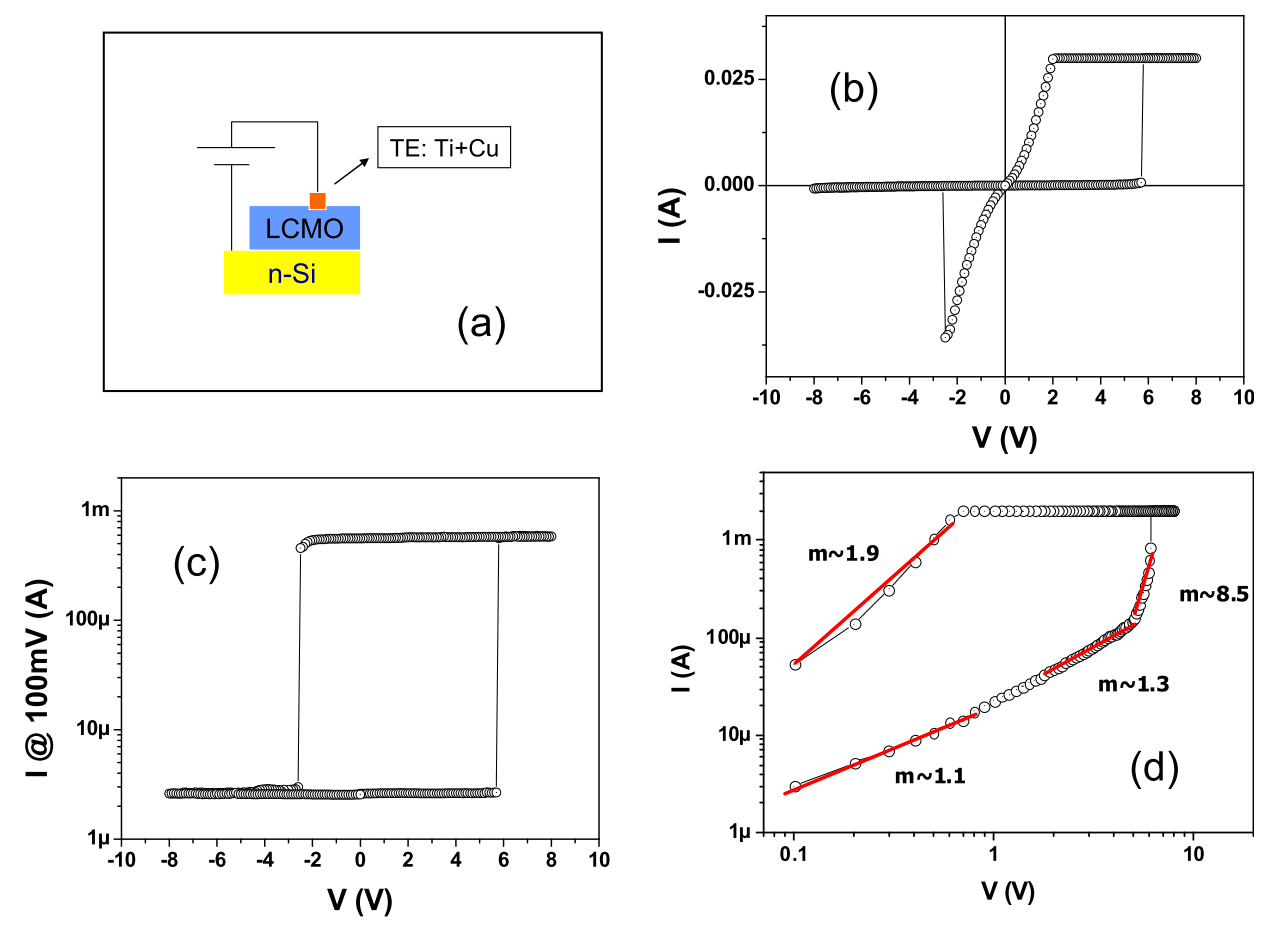

FIG. 1. (a) Sketch showing the electrode configuration and polarities used for the electrical characterization; (b) dynamic pulsed I-V curve corresponding to a $100 \mathrm{~nm}$ LCMO film. A $30 \mathrm{~mA}$ CC limited the current when the SET took place; (c) corresponding hysteresis switching loop, recorded simultaneously to the I-V curve; (d) positive voltage region of the $\mathrm{I}-\mathrm{V}$ curve in a log-log scale.
(RESET process). We have found a fairly symmetric behavior between the positive and negative regions of the I-V curve, indicating that the presence of the native ultrathin $\mathrm{SiO}_{\mathrm{x}}$ layer at the silicon (n-type)/manganite (p-type) interface prevents the formation of a $p-n$ junction and the appearance of a rectifying behavior. ${ }^{22}$ Figure 1(c) displays the corresponding HSL. One can see two well defined resistance states of $\sim 170 \Omega$ and $\sim 38 \mathrm{k} \Omega$, which gives an ON/OFF ratio of $\sim 220$. We tested the stability of the HSLs against repeated cycling at a fixed SET CC, and we found that $\mathrm{R}_{\mathrm{HIGH}}$ and $\mathrm{R}_{\mathrm{LOW}}$ remain reasonably stable for 70 consecutive loops. ${ }^{20}$ The squared shape of the HSL suggests the existence of only one active interface (plausibly the metal/oxide one, according to previously reported results in manganite samples with different metallic electrodes ${ }^{23}$ ); otherwise, a more complex shape such as the so-called "table with legs" would be expected. ${ }^{24,25}$ Figure 1(d) displays the positive stimulus branch of the I-V curve in a log-log scale. It is found that the HRS displays an ohmic behavior $(\mathrm{I} \propto \mathrm{V})$ for low voltages $(\mathrm{V}<1 \mathrm{~V})$, followed by a $\mathrm{I} \propto \mathrm{V}^{(1.3)}$ regime for intermediate voltages and a steep increase region $\left(\mathrm{I} \propto \mathrm{V}^{(8.5)}\right)$ for voltages close to the resistive transition to LRS. On the other hand, for this $30 \mathrm{~mA}$ SET CC, the LRS follows a $\mathrm{I} \propto \mathrm{V}^{(2)}$ law that could indicate the presence of a space-charge-limited current (SCLC) conduction mechanism. ${ }^{26}$ We have seen that both HRS and LRS are strongly dependant on the SET CC used on each I-V cycle. Figure 2(a) shows the evolution of $\mathrm{R}_{\mathrm{HIGH}}$ and $\mathrm{R}_{\mathrm{LOw}}$ as a function of $\mathrm{CC}$ for a top electrode area of $0.196 \mathrm{~mm}^{2}$, while Figure 2(b) displays the corresponding ON/OFF ratio. It is found that initially $\mathrm{R}_{\mathrm{HIGH}}$ remains nearly constant as $\mathrm{CC}$ increases, while $\mathrm{R}_{\mathrm{LOW}}$ decreases monotonically, increasing in this way the $\mathrm{ON} / \mathrm{OFF}$ ratio up to $\sim 400$ for $\mathrm{CC} \sim 90 \mathrm{~mA}$. For higher $\mathrm{CC}$ values, $\mathrm{R}_{\mathrm{HIGH}}$ starts to drop pronouncedly, the ratio ON/OFF decreasing concomitantly by almost two orders of magnitude. This behavior is qualitatively similar (but considerably more stable) when compared to the case of LCMO films with Ag top electrodes. $^{21}$
We consistently reproduced the electrical behavior for low CCs by using the Voltage Enhanced Oxygen Vacancies drift (VEOV) model introduced in Ref. 25. This model assumes an interface-type RS mechanism where the electrical transport across the sample is spatially inhomogeneous and takes place through several parallel conduction paths. The paths are assumed to be randomly distributed, hence producing a conductance proportional to the area. Within the VEOV model, since the paths are similar, one may model their typical behavior with a single resistor network, which is assumed to be one-dimensional for simplicity. Each element of the resistor network is meant to represent a small domain of the conductive path of nanometric size that is characterized by a local density of oxygen vacancies. In transition metal oxides, the resistivity is severely affected by the local oxygen stoichiometry. Hence, the model assumes that the resistivity of each nanodomain $\rho_{\mathrm{i}}$ is proportional to the local density of oxygen vacancies $\delta_{\mathrm{i}}$ according to $\rho_{\mathrm{i}}=\mathrm{A}_{\alpha} \delta_{\mathrm{I}}$, with $\alpha=\mathrm{I}$ or B corresponding to interface or bulk nanodomains, respectively. The proportionality between $\rho$ and $\delta$ follows from the assumption that oxygen vacancies disrupt the conduction through the Mn-O-Mn chains. The resistivity of the nanodomains close to the metal/oxide interface is much higher that of those located at bulk regions, due to the formation of a potential barrier at the metal/oxide interface, implying that $\mathrm{A}_{\mathrm{I}} \gg \mathrm{A}_{\mathrm{B}}{ }^{25}$ The application of electrical stress induces the migration of oxygen vacancies though the network according to the equation

$$
p_{i, i+1}=\delta_{i}\left(1-\delta_{i+1}\right) \exp \left(-V_{0, i}+\Delta V_{i}\right)
$$

which determines the oxygen vacancy transfer probability between adjacent sites. $\mathrm{V}_{0, \mathrm{i}}(\mathrm{i}=\mathrm{B}$ and $\mathrm{I})$ are dimensionless constants related to the activation energies for vacancies diffusion both in bulk and interfacial sites, respectively. $\Delta V_{\mathrm{i}}$ are the local potential drops due to applied electrical pulses, which are easily calculated from the 1D resistor network. 

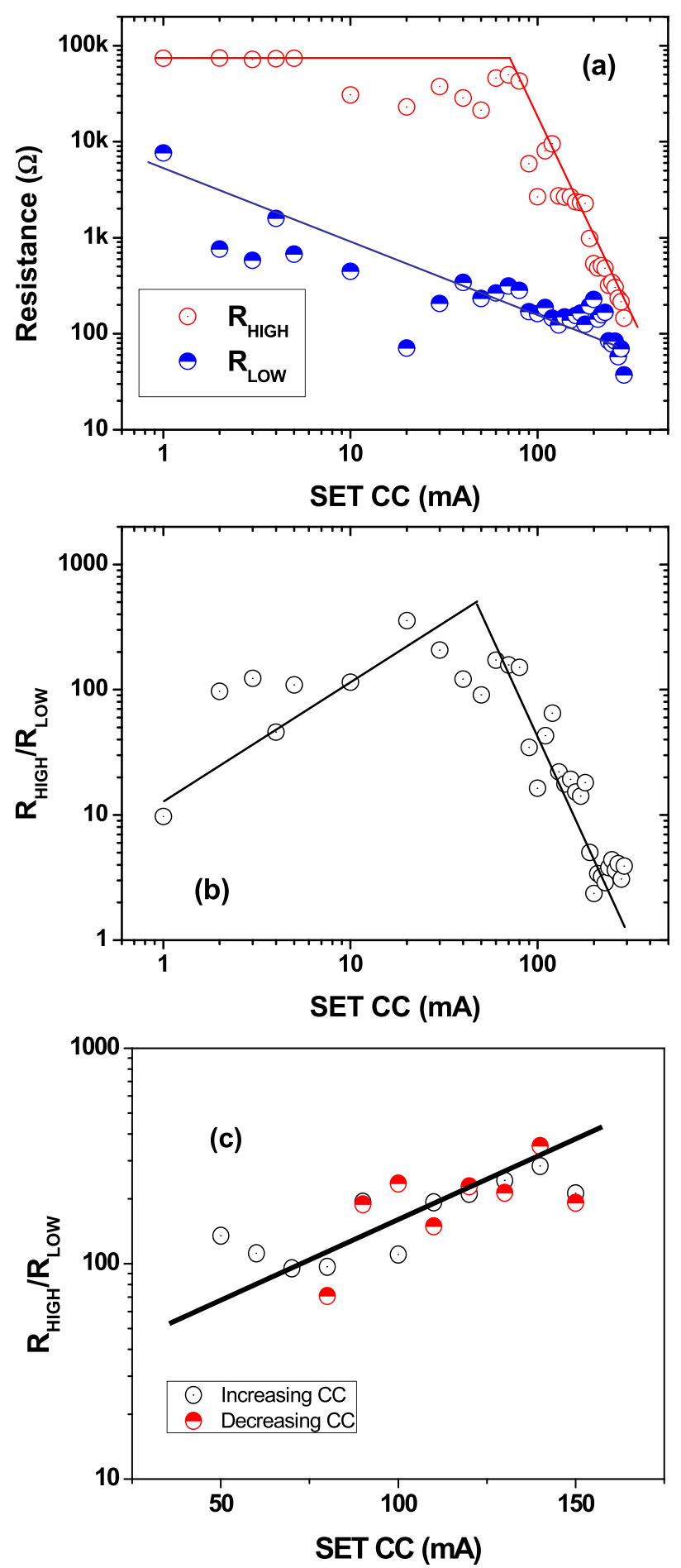

FIG. 2. (a) Evolution of $\mathrm{R}_{\mathrm{HIGH}}$ and $\mathrm{R}_{\mathrm{LOw}}$ as a function of the SET CC for a $100 \mathrm{~nm}$ LCMO film with a $0.196 \mathrm{~mm}^{2}$ top electrode; (b) $\mathrm{R}_{\mathrm{HIGH}} / \mathrm{R}_{\mathrm{LOw}}$ ratio as a function of the SET CC for the same film; (c) $\mathrm{R}_{\mathrm{HIGH}} / \mathrm{R}_{\mathrm{LOW}}$ ratio as a function of the SET CC for a thicker $250 \mathrm{~nm}$ LCMO film. We see a symmetric behavior both for increasing and decreasing $\mathrm{CC}$ (indicating the absence of degradation).

For positive (negative) electrical stimulus, vacancies migrate from the interface (bulk) to the bulk (interface) nanodomains, reversibly changing in this way the overall resistance of the device. The behavior of the model is simulated numerically, by performing a discretization of the time in time-steps. The transfers of oxygen vacancies and the local resistivity of each domain are computed and updated at every time-step. To apply the model to thin films geometry we assumed $\mathrm{N}_{\mathrm{I}}=\mathrm{N}_{\mathrm{B}}=10$ (the interface and the bulk regions present similar size, in contrast to the assumption $N_{B} \gg N_{I}$ used for ceramic samples ${ }^{25}$ ), $V_{0, I}=16, V_{0, B}=4, A_{I}=10000$ (a.u.), $\mathrm{A}_{\mathrm{B}}=0.5$ (a.u.), and $\delta_{0}=5 \times 10^{-2}$ (a.u.) (this value is higher than in Ref. 25 and consistent with the high oxygen deficiency present in our films). We found that this choice of model parameter captures the experimental behavior qualitatively. We calculated the evolution of the resistance of a single conducting path (which, besides a scaling factor, is representative of the behaviour of the overall device resistance) under voltage cycling, for different SET CCs. Figure 3(a) displays a calculated HSL for a $\mathrm{CC}=0.3$ a.u., showing that different non-trivial features of the
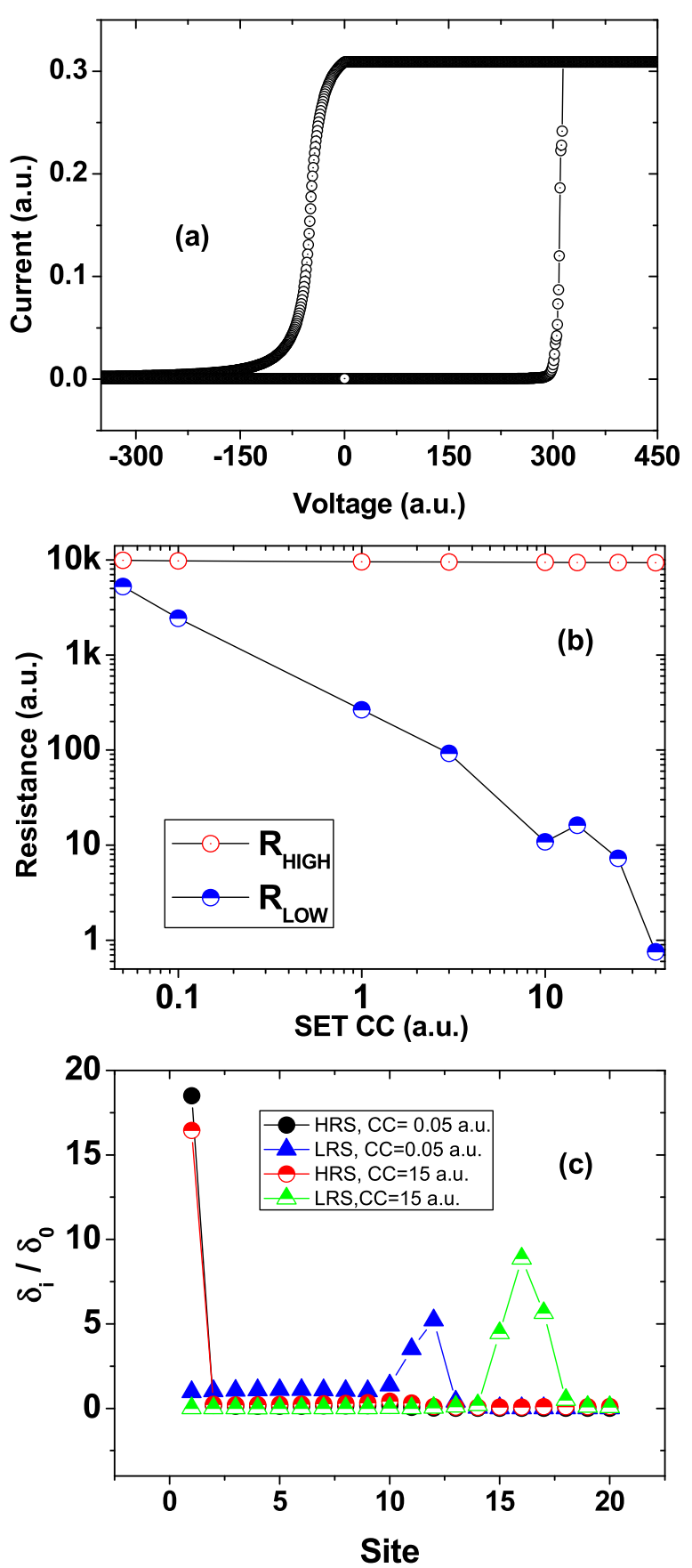

FIG. 3. (a) Calculated HSL for a SET CC of 0.3 a.u. by using the VEOV drift model; (b) calculated $\mathrm{R}_{\mathrm{HIGH}}$ and $\mathrm{R}_{\mathrm{LOW}}$ as a function of the SET CC; (c) calculated oxygen vacancy profiles of both LRS and HRS for different SET CCs. 
experimental electrical response such as the asymmetric SET and RESET voltages, or the HSL squared shape with sharp SET and RESET transitions are qualitatively described by the model. The evolution of the calculated $\mathrm{R}_{\mathrm{HIGH}}$ and $\mathrm{R}_{\mathrm{LOW}}$ as a function of the SET CC is depicted in Figure 3(b). We find that the calculated behaviour mimics the experimental one for $\mathrm{CC}<90 \mathrm{~mA}$, reinforcing the idea that the mechanism behind the observed RS for low CCs is consistent with the VEOV proposed model. Figure 3(c) displays the evolution of the vacancy profiles as the CC is increased. It is found that upon increasing the $\mathrm{CC}$ the number of oxygen vacancies moving from the interface to the bulk sites (SET process) increases, leading to a lowering of the LRS (we recall that the number of vacancies at the interface dominates the channel resistance as $A_{I} \gg A_{B}$ ). These vacancies are almost completely put back into the interface during the RESET process; thus, the HRS (and the corresponding vacancy profiles) remains roughly constant for different $\mathrm{CCs}$ as seen in Figures 3(b) and 3(c). For higher CCs, the collapse of the HRS is not captured by the VEOV model, suggesting that another RS mechanism becomes dominant in this regime. Indeed, going back to the experimental results, we see a change in the LRS conduction mechanism as the SET CC is increased. Figure 4(a) shows the evolution of the LRS parameter $m\left(\mathrm{I} \propto \mathrm{V}^{\mathrm{m}}\right.$, extracted from the slope of I vs. $\mathrm{V}$ in a $\log -\log$ graph) as CC increases. Figure 4(a) displays that
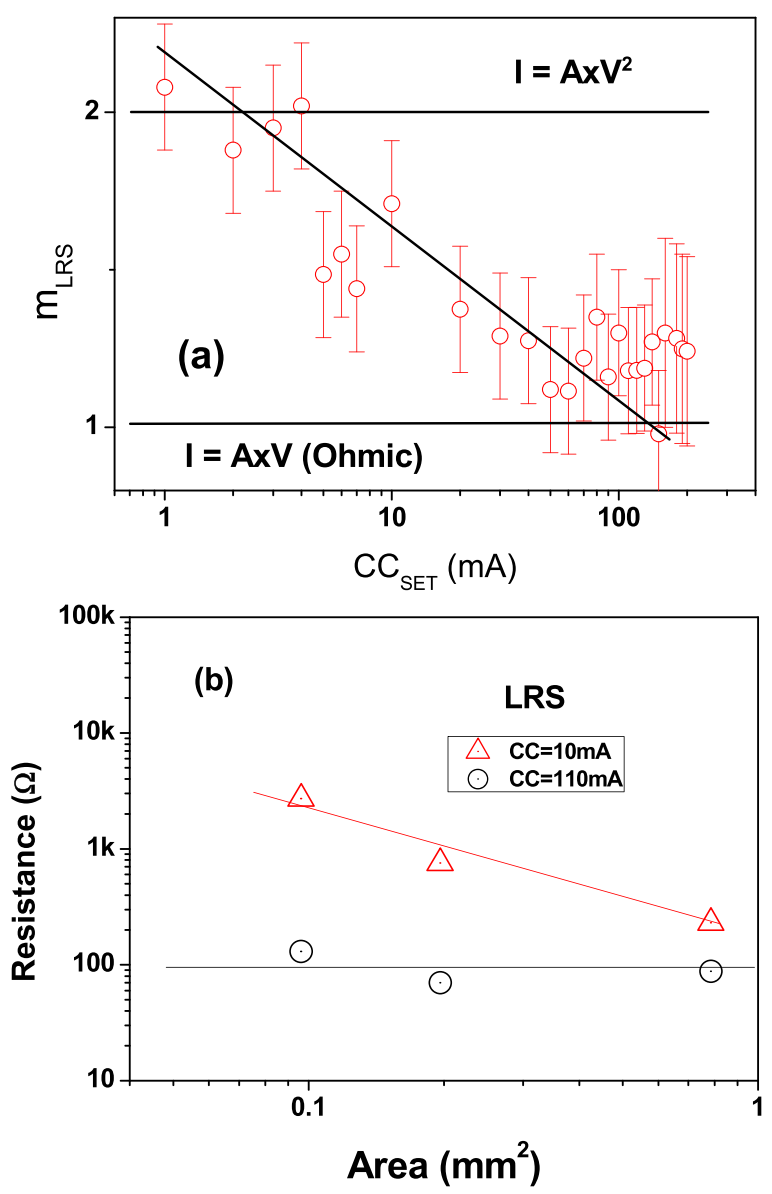

FIG. 4. (a) Evolution of the LRS I vs. V slope $m$ as a function of the SET CC. The error bars in $m$ are related to the dispersion found at fixed CC for different devices; (b) LRS as a function of the top electrode area, for SET CCs of $10 \mathrm{~mA}$ and $110 \mathrm{~mA}$. initially, for low CCs, $\mathrm{m}$ is $\sim 2$ (SCLC mechanism) and changes to $\mathrm{m} \sim 1$ (ohmic conduction, see also Ref. 20) as the $\mathrm{CC}$ increases. This behavior is consistent with a change from an interfacial-like resistive switching mechanism for low $\mathrm{CCs}$ to the onset of a filamentary regime for higher CCs. This finding is supported by Figure 4(b), displaying the evolution of the LRS as a function of the electrode area and for $\mathrm{CC}=10 \mathrm{~mA}$ and $110 \mathrm{~mA}$. It is found that for low CC the LRS clearly decreases around one order of magnitude, from $\sim 3 \mathrm{k} \Omega$ to $\sim 200 \Omega$, as the electrode area increases, which is the typical behaviour of interfacial-related RS. ${ }^{1}$ This is consistent with the presence of multiple conducting paths distributed on the surface of the device, with their resistance modulated by the local concentration of oxygen vacancies at the metal-oxide interface, which are the main assumptions made in the VEOV model described before. On the other hand, for high CC the area scaling breaks down as the LRS remains nearly constant around $100 \Omega$, suggesting that a single conductive (metallic) filament dominates the conduction. ${ }^{1}$ The development of this filament could be associated to the electrical-field induced diffusion of the metallic top electrode through the oxide until a conductive bridge connecting both electrodes is formed. Indications of filament formation have indeed been observed in other perovskite oxides such as $\mathrm{SrTiO}_{3}$ with Ti electrodes. ${ }^{27}$ Within this scenario, one can speculate that the formation of the metallic filament can be prevented if thicker films and lower electrical fields are used. We have tested this possibility by growing a $250 \mathrm{~nm}$ thick film, which was electrically stressed in the same conditions (and therefore under lower electrical fields) than the previous $100 \mathrm{~nm}$ film. Figure 2(c) shows the evolution of the ON/OFF ratio as a function of the SET CC for the $250 \mathrm{~nm}$ film, displaying a monotonic increase of the ratio up to $\mathrm{CC}=150 \mathrm{~mA}$. This is in contrast to the $100 \mathrm{~nm}$ film case, where a clear degradation of the ON/OFF ratio was observed from $\mathrm{CC} \sim 90 \mathrm{~mA}$ (as seen in Figure 2(b)). This indicates that we may indeed prevent the onset of the single-filament regime by making the oxide thicker. This is an important issue when considering possible applications, since filament-based resistive switching is usually thought as less reliable than interfacial-related effects, and therefore strategies to keep the device working in an interface-related regime are welcome.

In summary, we have shown that low cost $\mathrm{n}-\mathrm{Si} / \mathrm{La}_{2 / 3} \mathrm{Ca}_{1 / 3} \mathrm{MnO}_{3} / \mathrm{M}(\mathrm{M}=\mathrm{Ti}+\mathrm{Cu})$ devices present a reliable resistive switching behavior. $\mathrm{R}_{\mathrm{HIGH}}, \mathrm{R}_{\mathrm{LOW}}$ and therefore the ON/OFF ratio display a strong dependence on the SET CC, reaching a maximum $\sim 400$ for $C C \sim 90 \mathrm{~mA}$. A regime change between two resistive switching mechanisms when the CC is increased has been identified. For low CCs, the observed resistive switching mechanism is related to the migration of oxygen vacancies close to the manganite/metal interface. This result is supported by numerical simulations using the VEOV drift model. For higher CCs, the onset of a regime dominated by the conduction of a single-filament, which would account for the observed collapse of the $\mathrm{ON} / \mathrm{OFF}$ ratio was suggested. We argue that this regime is likely related to the migration of atoms from the metal electrode. Finally, we proposed and tested a simple strategy to prevent the onset of the filamentary regime. 
We acknowledge financial support from UNSAM (project SJ10/05), MINCYT (INNOVAR program), and CONICET (PIPs 047 and 291). We thank F. Saccone (FIUBA) for the use of the PLD facility.

${ }^{1}$ A. Sawa, Mater. Today 11, 28 (2008).

${ }^{2}$ R. Waser, R. Dittmann, G. Staikov, and K. Szot, Adv. Mater. 21, 2632 (2009).

${ }^{3}$ D. B. Strukov and H. Kohlstedt, MRS Bull. 37, 108 (2012).

${ }^{4}$ D. S. Shang, Q. Wang, L. D. Chen, R. Dong, X. M. Li, and W. Q. Zhang, Phys. Rev. B 73, 245427 (2006).

${ }^{5}$ Y. B. Nian, J. Strozier, N. J. Wu, X. Chen, and A. Ignatiev, Phys. Rev. Lett. 98, 146403 (2007).

${ }^{6}$ K. Szot, W. Speier, G. Bihlmayer, and R. Waser, Nature Mater. 5, 312 (2006).

${ }^{7}$ W. Shen, R. Dittmann, U. Breuer, and R. Waser, Appl. Phys. Lett. 93, 222102 (2008)

${ }^{8}$ H.-S. Lee, S.-G. Choi, H.-H. Park, and M. J. Rozenberg, Sci. Rep. 3, 1704 (2013).

${ }^{9}$ S. H. Chang, J. S. Lee, S. C. Chae, S. B. Lee, C. Liu, B. Khang, D. W. Kim, and T. W. Noh, Phys. Rev. Lett. 102, 026801 (2009).

${ }^{10}$ S. Jin, T. H. Tiefel, M. McCormack, R. A. Fastnacht, R. Ramesh, and L. H. Chen, Science 264, 413 (1994).

${ }^{11}$ A. Asamitsu, Y. Tomioka, H. Kuwahara, and Y. Tokura, Nature 388, 50 (1997).

${ }^{12}$ S. Q. Liu, N. J. Wu, and A. Ignatiev, Appl. Phys. Lett. 76, 2749 (2000).

${ }^{13}$ M. Quintero, A. G. Leyva, and P. Levy, Appl. Phys. Lett. 86, 242102 (2005).

${ }^{14}$ N. Das, S. Tsui, Y. Y. Xue, Y. Q. Wang, and C. W. Chu, Phys. Rev. B 78, 235418 (2008).
${ }^{15}$ Ch. Jooss, J. Hoffmann, J. Fladerer, M. Ehrhardt, T. Beetz, L. Wu, and Y. Zhu, Phys. Rev. B 77, 132409 (2008).

${ }^{16}$ N. A. Tulina, S. A. Zverkov, Y. M. Mukovskii, and D. A. Shulyatev, Europhys. Lett. 56, 836 (2001).

${ }^{17}$ J. O. Krisponeit, C. Kalkert, B. Damaschke, V. Moshnyaga, and K. Samwer, Phys. Rev. B 82, 144440 (2010).

${ }^{18}$ S. M. Sadaf, E. M. Bourim, X. Liu, S. H. Chodhury, D. W. Kim, and H. Hwang, Appl. Phys. Lett. 100, 113505 (2012).

${ }^{19}$ M. Fujimoto, H. Koyama, Y. Nishi, and T. Suzuki, Appl. Phys. Lett. 91, 223504 (2007).

${ }^{20}$ See supplementary material at http://dx.doi.org/10.1063/1.4826484 for additional figures.

${ }^{21}$ I. Alposta, A. Kalstein, N. Ghenzi, S. Bengió, G. Zampieri, D. Rubi, and P. Levy, IEEE Trans. Magn. 49, 4582 (2013).

${ }^{22}$ Assuming a native $\mathrm{SiO}_{\mathrm{x}}$ thickness of $\sim 10 \AA$ and a dielectric strength of 5 $\times 10^{6} \mathrm{~V} / \mathrm{cm}$ (Klein and Gafni (1966)), a voltage of $\sim 500 \mathrm{mV}$ is enough to permanently break this ultrathin layer. This voltage is more than an order of magnitude lower that the observed SET voltage ( $\sim 6 \mathrm{~V})$, strongly indicating that when our first SET process takes place the native $\mathrm{SiO}_{2}$ layer is already dielectrically broken, and the observed resistive switching originates at the metal/manganite interface, as proposed.

${ }^{23}$ R. Yang, X. M. Li, W. D. Yu, X. D. Gao, D. S. Shang, X. J. Liu, X. Cao, Q. Wang, and L. D. Chen, Appl. Phys. Lett. 95, 072105 (2009).

${ }^{24}$ M. Quintero, P. Levy, A. G. Leyva, and M. J. Rozenberg, Phys. Rev. Lett. 98, 116601 (2007).

${ }^{25}$ M. J. Rozenberg, M. J. Sánchez, R. Weht, C. Acha, F. Gomez-Marlasca, and P. Levy, Phys. Rev. B 81, 115101 (2010).

${ }^{26}$ A. Rose, Phys. Rev. 97, 1538 (1955).

${ }^{27}$ S. Stille, Ch. Lenser, R. Dittmann, A. Köhl, I. Krug, R. Muenstermann, J. Perlich, C. M. Schneider, U. Klemradt, and R. Waser, Tech. Dig. Front. Electron. Mater. 2012, 665. 